\title{
Avaliação externa de organizações hospitalares no Brasil: podemos fazer diferente?
}

\author{
External evaluation of hospital organizations in Brazil: \\ could things be done differently?
}

\footnotetext{
${ }^{1}$ Instituto Sírio-Libanês de Ensino e Pesquisa. R. Cel Nicolau dos Santos 69, Bela Vista. 01308-060 São Paulo SP Brasil.

lauschi@uol.com.br
}

\begin{abstract}
This paper addresses different aspects related to the use of external evaluation models of health services in Brazil, especially hospital accreditation. It is based upon the author's experience in implementing quality evaluation models in hospitals, as well as on her master and PhD works. The author presents a few aspects concerning the impact of the use of these models in hospitals and the specificities of their application. The results are listed: management enhancement, standardization of processes, personnel training, teamwork, patient-focused care, motivation, external recognition, culture change. The author points out the sundry aspects concerning the way such models are used in Brazilian hospitals.
\end{abstract}

Key words Health evaluation, Service evaluation, Quality of healthcare, Accreditation
Resumo Este artigo aborda aspectos relacionados ao uso de modelos de avaliação externa de serviços de saúde no Brasil, sobretudo acreditação hospitalar, a partir da experiência da autora com a implementação de modelos de avaliação da qualidade em hospitais, bem como de sua reflexão sobre acreditação enquanto objeto de estudo da pós-graduação. A autora aponta alguns dos resultados do uso destes modelos em hospitais e as especificidades de sua aplicação. Esses resultados são enumerados: aprimoramento da gestão, padronização de processos, treinamento de pessoal, trabalho em equipe, atenção focada no paciente, motivação, reconhecimento externo, mudança de cultura. A autora problematiza o modo como tais modelos têm sido empregados nos hospitais brasileiros.

Palavras-chave Avaliação em saúde, Avaliação de serviços, Qualidade da assistência, Acreditação 


\section{Modelos de avaliação externa da qualidade}

Hoje, poucos ousariam se posicionar contra a transparência da gestão, da vida organizacional e dos resultados dos serviços de saúde. Isto explicaria a pertinência do uso da avaliação externa como instrumento de gestão e sua crescente popularidade.

No final dos anos 90, poucos hospitais ou profissionais de saúde sabiam o que era acreditação hospitalar, certificação pelas normas ISO, prêmio nacional da qualidade ou ainda sistemas de monitoramento do desempenho dos serviços de saúde no Brasil ${ }^{1}$. Hoje, anos depois, o cenário mudou $^{2,3}$. Não estamos certamente diante do que alguns imaginaram ou sonharam, mas há fatos que merecem destaque: diferentes modelos de acreditação hospitalar coexistem em nosso país - Organização Nacional de Acreditação (ONA), Joint Commission International (JCI), Acreditação canadense, National Integrated Accreditation for Healthcare Organizations (NIAHO); diversos hospitais são hoje acreditados; um número crescente de serviços de saúde é hoje certificado pelas normas ISO 9.001, 14.000, 31.000 e OHSAS 18.001; as boas práticas de gestão preconizadas pelo Prêmio Nacional da Qualidade e pelas escolas de gestão são conhecidas e por vezes utilizadas.

Algumas discussões da década de 90 relacionadas às diferenças existentes entre os modelos de avaliação externa da qualidade ou ainda a busca pelo modelo mais adequado para a saúde não fazem mais sentido. A acreditação ganhou espaço neste contexto, as normas ISO têm hoje indicação mais precisa e os prêmios da qualidade são utilizados em contextos específicos.

Cada modelo desenvolveu sistema de avaliação próprio, ainda que as últimas versões de todos os instrumentos contenham muitas modificações que apontam maior similaridade. Os termos evidenciam estas diferenças - avaliação inicial, visita de acreditação, auditoria, auditoria interna, autoavaliação, relatório de candidatura, etc. ${ }^{4-6}$. Para quem já "sofreu" diferentes tipos de avaliação, a afiliação do avaliador pode muitas vezes ser identificada de forma rápida, dada a sua postura e atitude. Um aspecto que parece favorecer a manutenção dos padrões desenvolvidos é a periodicidade das avaliações, entremeadas por momento de reflexão interna - auditoria interna, autoavaliação, etc.

As especificidades se confundem em parte com o ponto alto de cada modelo. A acreditação propõe padrões inerentes às atividades assistenciais, sobretudo as vertentes norte-americanas, com grande ênfase na coordenação dos cuidados e dos cuidadores. O prontuário do paciente tem papel preponderante e é instrumento precioso para se avaliar a qualidade dos cuidados. Aspectos considerados prioritários para aumentar a segurança do cuidado foram incorporados de forma explícita por quase todos os modelos de acreditação, bem como os indicadores da qualidade da assistência ${ }^{4,5}$.

A certificação ISO utiliza a "análise crítica" pela direção como estratégia de reflexão dos gestores sobre o sistema da qualidade, com o uso de informações e indicadores. Esta análise orienta a decisão gerencial sobre as ações prioritárias. Outro aspecto introduzido originalmente por estas normas refere-se, sobretudo, à gestão dos riscos, mais especificamente o monitoramento das "não conformidades", das "ações corretivas" e "preventivas". A rastreabilidade dos produtos é outro elemento muito peculiar da norma e foi ultimamente valorizado na saúde em geral, sobretudo com a crescente importância da segurança. A gestão da documentação institucional ocupa posição de destaque nestas normas?

Os modelos relacionados aos prêmios foram os primeiros a abordar enfaticamente a liderança institucional. A noção de responsabilidade social e de coletividade é critério específico deste modelo. O monitoramento de indicadores, sobretudo de desempenho, é há muito preconizado ${ }^{8}$.

Observa-se o crescimento de iniciativas aparentemente paralelas no seio de cada modelo. É o caso da certificação de doenças ou programas da acreditação da Joint Commission, ou ainda as metas internacionais de segurança, os eventos sentinela e as medidas do desempenho, iniciativas já mais antigas ${ }^{5}$. No caso dos prêmios - os grupos temáticos ou "comunidades de prática" ou ainda os frameworks. Estas iniciativas viriam reforçar os sistemas existentes ou ao contrário, seriam elas esforços para criar novas alternativas a modelos hoje um pouco desgastados?

\section{Resultados associados a estas iniciativas}

Uma vez que a intenção inicial influencia o modo de aplicação destes modelos e seu sucesso, pode-se dizer que os resultados são muito variáveis. De forma geral pode-se dizer que para ser acreditado ou certificado, é preciso respeitar os padrões propostos. Deste modo, é frequente observar que as instituições que seguem tais modelos empregam seus jargões, instrumentos e estratégias associadas, sem o que não seria possível es- 
tar "conforme". Enorme é a diversidade existente na sua aplicação, daí a grande variabilidade dos resultados encontrados ${ }^{10}$. Pode-se dizer, no que tange a acreditação hospitalar, que a organização acreditadora tem influência importante sobre este fenômeno, sobretudo no caso da acreditação da ONA, realizada por intermédio de vários parceiros devidamente credenciados.

Alguns resultados tidos como positivos são ${ }^{1}$ : Aprimoramento da gestão: estes modelos introduziram, de diferentes maneiras, a ideia do planejamento estratégico como instrumento de gestão e, ao mesmo tempo, de sobrevivência num contexto externo de grande dinamismo. A necessidade de instrumentos mais precisos para a gestão, sobretudo de dados quantitativos, privilegia o uso de indicadores para orientar a tomada de decisão.

$\checkmark \quad$ Padronização dos processos: de modo geral, o uso destes modelos permite explicitar os padrões esperados para processos considerados prioritários e/ou críticos e assim aos poucos reduzir a variação de certos processos e das práticas clínicas e administrativas relacionadas. Normas, regulamentos, orientações, procedimentos ou documentos técnicos são produzidos com o intuito de homogeneizar as ações passíveis de padronização. Protocolos ou itinerários clínicos, amplamente estimulados pelos sistemas de acreditação, têm sido desenvolvidos.

$\checkmark$ Treinamento do pessoal: o desenvolvimento ou aprimoramento de competências é preocupação central de toda iniciativa da qualidade. Assim sendo, hospitais envolvidos com estes modelos tendem a aumentar a oferta de cursos, levando em muitos casos ao aumento do número de horas de treinamento por colaborador. $\mathrm{O}$ planejamento dos treinamentos e seu monitoramento também recebem grande impulsão ${ }^{11}$.

$\checkmark$ Trabalho em equipe: o bom funcionamento da equipe multiprofissional e interdisciplinar em torno da assistência ao paciente é preocupação constante destes e, ao que parece, permanece grande desafio e por vezes mesmo um sonho... Estas iniciativas têm frequentemente o mérito de sensibilizar as instituições e seus dirigentes para a importância do tema.

$\checkmark \quad$ Cuidado focado no paciente: Muitas instituições estão hoje mais atentas às expectativas e necessidades dos pacientes, ainda que tenham dificuldade em colocá-los no centro de suas preocupações. A informação hoje à disposição do paciente é quantitativa e qualitativamente superior ao que antes existia, muito embora ainda haja muita margem para melhorias. A maior par- te dos modelos de acreditação enfatiza os direitos dos pacientes, entre os quais a participação na tomada de decisão. Todos estes modelos reiteram a importância da mensuração e aprimoramento da satisfação do usuário.

$\checkmark \quad$ Motivação: num momento inicial, todos estes modelos podem motivar parte importante dos trabalhadores, sobretudo quando a busca por melhoria da qualidade se faz de forma voluntária e num ambiente agradável. A dificuldade é manter essa motivação depois da avaliação externa e não são raros os casos de "traumatismo institucional" relacionados a este tipo de experiência ou de "depressão pós acreditação"... 12,13

$\checkmark \quad$ Reconhecimento externo: é muitas vezes a razão inicial de se buscar um ou outro tipo de avaliação externa. De modo geral, ser um hospital "acreditado", "certificado" ou ainda ganhador de prêmio nacional da qualidade demonstra esforço importante em ser reconhecido por especialistas. Ainda que boa parte da população não saiba ao certo o que isto significa, muitos usuários estão hoje atentos a "detalhes" que os auxiliem na escolha de um hospital e não de outro. Algumas operadoras começaram, apenas recentemente, a reconhecer o esforço dos estabelecimentos acreditados por meio de pagamento diferenciado.

$\checkmark \quad$ Mudança da cultura: quem participa deste tipo de iniciativa institucional bem conduzida, não tem muita dificuldade para constatar que houve sim mudança no comportamento das pessoas e que muitos processos foram modificados ${ }^{11}$. No entanto, manter o mesmo ritmo, uma vez terminada a primeira empreitada, é tarefa árdua.

Os resultados citados foram apontados por alguns estudos que envolveram número limitado de hospitais e que utilizaram metodologias qualitativas. Ou seja, estes achados não podem, ao menos por enquanto, ser generalizados ${ }^{1,13,14}$.

\section{Nem tudo é perfeito...}

Apesar de termos até então falado dos modelos como se cada um fosse uma entidade clara e homogênea, este não é o caso. No que se refere à acreditação, muito embora exista hoje convergência importante dos modelos no nível internacional, cada organismo guarda suas práticas de avaliação, suas interpretações para determinado tema, seu sistema de pontuação, sua classificação dos resultados. A dinâmica criada numa determinada realidade - por exemplo, a "aura" ou imagem dos modelos numa cidade, região ou país são muitas vezes reveladoras do modo de se acreditar ou de se certificar ou de se concorrer a 
prêmio... Algumas regras excessivas acabam por desencadear reações ou adaptações para "contornar" o estabelecido ${ }^{15}$. Isto parece natural em algumas culturas, mas considerando que o mesmo fenômeno pode ser observado em várias culturas distintas, pode-se especular que se trata de fenômeno quase mundial...

No que se refere às normas ISO, dada a dificuldade inicial dos profissionais da saúde em entender a nova terminologia e, por outro lado, dos "normistas" em entender a saúde, um mesmo item da norma foi traduzido de modo desigual nos diferentes países ${ }^{10}$. O mesmo ocorre com os vários prêmios, embora eles, assim como os demais, guardem aspectos similares nos diferentes países.

Qualidade é palavra de difícil definição. Excelência, ainda pior... E, para completar, todos estes vocábulos integram hoje o palavreado vazio de muitos burocratas ou políticos, ao mesmo tempo em que guardam seu valor nas esferas em que sua importância é reconhecida.

O grande "boom" da qualidade ajudou a convencer muita gente sobre sua importância. No entanto, as inúmeras dificuldades para progredir dentro da busca por melhor qualidade da assistência fazem com que muitos desanimem ao longo desta empreitada. Até hoje poucas instituições são capazes de dizer quanto foi gasto com esta ou aquela iniciativa e quais os resultados concretos obtidos, inclusive financeiros. Poucas questionam a relação custo-benefício dos modelos de avaliação externa e a necessidade de mecanismos de regulação desta atividade voluntária e independente.

Alguns críticos destes modelos os consideram "esclerosados" ou então "esclerosantes", dados os efeitos causados, sobretudo no médio ou longo prazos, uma vez que muito frequentemente sua aplicação não respeita suficientemente as especificidades das organizações. Quando se trata de iniciativa imposta pela diretoria da organização, pela autoridade sanitária ou ainda pelas seguradoras, pouco espaço resta para a negociação. Aos poucos, os profissionais inicialmente entusiastas, convencidos da importância de melhorar as práticas assistenciais, desanimam e não mais acreditam no lado positivo da qualidade. Restam, assim, apenas aspectos que quando excessivos se tornam negativos: normalização, procedimentos, medidas, documentos, etc...

Além da desmotivação, o grande efeito deletério e perverso da aplicação destes modelos tem sido o declínio dramático da criatividade. Em muitas realidades, não há mais espaço para ou- tras iniciativas, para ideias inovadoras, para ser diferente...

Não se pode negar a necessidade e a importância de se ter qualidade na assistência, tampouco segurança crescente para os usuários e os profissionais. Mas não seria possível aumentar a qualidade e a segurança de nossas organizações de forma menos rígida e inflexível? Por que não adotar inicialmente estratégias para melhorar a qualidade de forma participativa, debaixo para cima, para depois fazer emergir entre os profissionais de saúde a necessidade de adoção de padrões externos?

\section{Para onde vamos?}

No Brasil, assistimos hoje à divulgação positiva das ideias contidas nestes modelos. Nas várias regiões, há organizações de saúde que os seguem. Nos eventos da área e nos cursos de gestão, o linguajar antes pouco familiar é amplamente empregado pelos profissionais de saúde. A sensação é de que estas boas ideias e práticas se instalaram em nosso meio e avançam de forma clara.

Apesar dos quase 15 anos de uso deste modelo no Brasil, somente algumas organizações têm hoje sistemas da qualidade maduros. De modo geral são estas iniciativas recentes, e não há estimativas de quantas destas foram bem sucedidas. Não é fácil determinar a contribuição exata destas práticas para os diferentes atores, sobretudo para o paciente. Fato é que alguns poucos hospitais dispõem de medidas exatas que apontam segurança crescente da assistência, bem como aumento constante da qualidade de seus serviços.

Até há pouco tempo, não se podia falar em obrigatoriedade do uso de tais modelos. Mais recentemente, com o Programa de Qualificação das Operadoras e dos Prestadores da Agência Nacional de Saúde, com a divulgação da portaria relacionada à Segurança do Paciente da Agência Nacional de Vigilância Sanitária, pode-se dizer que os padrões da qualidade da assistência e da segurança do paciente são hoje parte integrante da realidade da saúde brasileira e serão, aos poucos, reforçados... ${ }^{16,17}$ Bom seria aproveitar a multiplicidade de modelos aqui existentes e, ao mesmo tempo, aprender com o que ocorreu nos países que os utilizam há mais tempo...

Em várias realidades, o aumento das exigências em torno da melhoria do desempenho e da transparência da gestão dos hospitais, bem como da prática profissional tem levado à exaustão boa parte dos profissionais de saúde. Há algumas provas de que apesar dos esforços crescen- 
tes para aumentar a qualidade e a segurança das organizações de saúde, pouco se avançou em alguns domínios ${ }^{18}$. Assim sendo, muitos sistemas vivem hoje certo impasse, ainda que nem todos os atores estejam conscientes da gravidade deste fenômeno. Este panorama é o prenúncio de mudanças importantes e necessárias na gestão da qualidade. Para sobreviver, a qualidade da saúde, que mal desponta no Brasil, terá que ser reinventada... Reconciliar normas e liberdade, compatibilizar os extremos ou simplesmente aprender a gerenciar as diferenças de modo bem diferente...

\section{Considerações finais}

A contribuição dos modelos de avaliação externa, sobretudo da acreditação para os hospitais brasileiros é inquestionável. Apesar do número restrito de hospitais envolvidos em processos de melhoria da qualidade desta natureza, hoje boa parte das lideranças dos hospitais brasileiros sabe da existência destes modelos. Os hospitais com reconhecimento externo desenvolvem inúmeras atividades para garantir a manutenção dos padrões alcançados. No entanto, a sensação é de que boa parte dos profissionais da área aproximou-se do jargão e das ideias destes modelos, utiliza palavreado adequado, mas poucos implementaram efetivamente tais padrões, e raros são os que de fato conseguiram induzir mudança concreta em suas realidades no longo prazo.

Há hoje aplicação acrítica dos padrões externos, sem haver necessariamente adaptação para a realidade local e a cultura organizacional.

O cenário nacional é singular - oferta diversificada de modelos e possibilidade de escolher o mais adequado. No entanto, o uso dos mesmos tem forjado semelhanças crescentes no interior dos serviços, não somente para aspectos que garantam qualidade e segurança para o profissional e para o usuário, mas também para aspectos mais periféricos relacionados à visão de gestão e de processos de cada organização acreditadora. Em outras palavras, a margem para a criatividade e para a singularidade organizacional encontra-se em redução, o que ameaça não somente a motivação dos profissionais, mas sobretudo alguns aspectos positivos das especificidades da realidade organizacional e de sua cultura.

Que tal seguirmos modelos simplificados de avaliação externa da qualidade e darmos espaço para iniciativas com maior grau de autonomia e liberdade locais? 


\section{Referências}

1. Schiesari LMC. Resultados de iniciativas da qualidade em hospitais brasileiros [tese]. São Paulo: Universidade de São Paulo;2003.

2. Bonacim CAG, Araujo AMP. Avaliação de desempenho econômico-financeiro dos serviços de saúde: os reflexos das políticas operacionais no setor hospitalar. Cien Saude Colet 2011; 16(Supl. 1):1055-1068.

3. Tanaka O, Tamaki EM. O papel da avaliação para a tomada de decisão na gestão de serviços de saúde. Cien Saude Colet 2012; 17(4):821-828.

4. Instituto Qualisa de Gestão. Manual de Acreditação Internacional. Programa de Acreditação Canadense CCAP. Rio de Janeiro: IQG \& CCHSA International; 2007.

5. Joint Commission International. Padrões de Acreditação da Joint Commission Internacional para Hospitais. Rio de Janeiro: Consórcio Brasileiro de Acreditação de Sistemas e Serviços de Saúde (CBA); 2011.

6. Organização Nacional de Acreditação (ONA). Manual Brasileiro de Acreditação - Organizações Prestadoras de Serviços de Saúde. São Paulo: ONA; 2010.

7. Associação Brasileira de Normas Técnicas. NBR ISP 19011. Diretrizes para auditorias de sistema de gestão da qualidade e/ou ambiental. 2008.

8. Fundação Nacional da Qualidade (FNQ). Critérios de Excelência. 19a ed. São Paulo: FNQ; 2012.

9. European Foundation for Quality Improvement. EFQM Excellence model. 2010. [acessado 2014 ago 8]. Disponível em: http://www.efqm.org/the-efqm-excellence-model

10. Sweeney J, Heaton C. Interpretations and variations of ISO 9000 in acute health care. Int J Qual Health Care 2000; 12(3):203-209.

11. Greenfield D, Pawsey M, Braithwaite J. O papel e o impacto da acreditação na revolução da atenção à saúde. Revista Acreditação 2011; 1(2):11.

12. Degois JM, Gouin P, Pocthier J, Baudry V, Maguerez G. Comment éviter la "dépression postaccréditation"? L'expérience du centre hospitalier de Dreux. Gestions hospitalières 2002; (421):801-804.
13. Greenfield D, Braithwaite J. Health sector accreditation research: a systematic review. Int J Qual Health Care 2008; 20(3):172-183.

14. Ovretveit J. Integrated quality development in public healthcare: a comparison of six hospitals quality programmes and a practical theory for quality development. Oslo: Aslaksen, Norwegian Medical Association; 1999.

15. Gaulejac V. La société malade de la gestion. Paris: Editions du Seuil; 2005.

16. Agência Nacional de Saúde Suplementar (ANS). Qualificação dos Prestadores de Serviços - Qualiss. [acessado 2014 ago 8]. Disponível em: http://www.ans.gov.br/ espaco-dos-prestadores/qualiss

17. Brasil. Ministério da Saúde (MS). Agência Nacional de Vigilância Sanitária. RDC 36. Brasília : MS ; 1 de abril de 2013.

18. Michel P, Minodier C, Moty-Monnereau C, Lathelize M, Domecq S, Chaleix JL, Olier L. Les événements indésirables graves dans les établissements de santé: fréquence, évitabilité et acceptabilité. Etudes et resultants 761, 2011. [acessado 2014 ago 8]. Disponível em: http://www.google.com.br/url?sa=t\&rct=j\&q=\&es $\mathrm{rc}=\mathrm{s} \&$ source $=$ web $\& \mathrm{~cd}=1$ \&ved $=0 \mathrm{CB} 8 \mathrm{QFjAA} \& u r \mathrm{r}$ $=\mathrm{http} \% 3 \mathrm{~A} \% 2 \mathrm{~F} \% 2 \mathrm{Fwww}$.nosopicard.com $\% 2$ Fiso album\%2Fenquete_eneis_2110.pdf\&ei=9DvlU6KLBvO_sQThy4DQBA\&usg=AFQjCNEnOIaBbVaams43Ch81bDH9KxaqQQ \&sig2=wZsLIWsaMCuhZ7j0abPREw\&bvm=bv.72676100,d.cWc

Artigo apresentado em 30/08/2013

Aprovado em 27/10/2013

Versão final apresentada em 31/10/2013 